\title{
The Use of Triple Therapy in Chronic Obstructive Pulmonary Disease and Outcomes of the IMPACT Trial
}

\author{
An Expert Interiew with Mario Cazzola
}

University of Rome Tor vergata, Rome, Italy

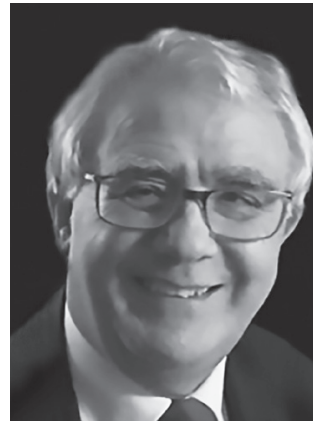

Mario Cazzola

Mario Cazzola is an Honorary Professor of Respiratory Medicine at the University of Rome Tor Vergata, Italy, and Visiting Professor at the Sackler Institute of Pulmonary Pharmacology, GKT School of Biomedical Sciences, London, UK. He is a fellow of the European Respiratory Society and has received the 2015 European Respiratory Society Lifetime Achievement Assembly Award.

\section{Keywords}

Chronic obstructive pulmonary disease, IMPACT study, COPD GOLD guidelines, triple therapy

\section{Disclosure: Mario Cazzola has participated as a speaker and advisor in scientific meetings and courses under the sponsorship of Almiral AstraZeneca, Biofutura, Boehringer Ingelheim, Chiesi Farmaceutici, GlaxosmithKline, Menarini Group, Lallemand, Mundipharma, Novartis, Pfizer, Verona Pharma, and Zambon, and is or has been a consultant to Chiesi Farmaceutici, Lallemand, Novartis, Verona Pharma, and Zambon. He is also a member of the journal's editorial board.}

Review Process: This is an expert interview and, as such, has not undergone the journal's standard peer review process.

Authorship: The named author meets the International Committee of Medical Journal Editors (ICMJE) criteria for authorship of this manuscript, takes responsibility for the integrity of the work as a whole, and has given final approval for the version to be published.

open Access: This article is published under the Creative Commons Attribution Non-commercial License, which permits any non-commercial use, distribution, adaptation, and reproduction provided the original author and source are given appropriate credit. () The Author 2018 Received: 11 July 2018

Published Online: 5 September 2018

Citation: European Respiratory \& Pulmonary Diseases. 2018;4(1):17-8

Corresponding Author: Mario Cazzola Dipartimento di Medicina dei Sistemi, Università di Roma Tor Vergata, Via Montpellier 1, Rome 00133, Italy. E: mario.cazzola@uniroma2.it

Support: No funding was received in the publication of this article.
$T$ riple inhaled therapy for chronic obstructive pulmonary disease (COPD) comprises the combination of an inhaled corticosteroid (ICS), a long-acting $\beta 2$-agonist (LABA) and a long-acting muscarinic antagonist (LAMA). The use of triple therapy is recommended by the Global Initiative for Chronic Obstructive Lung Disease (GOLD) for patients who experience recurrent exacerbations despite treatment with either a dual bronchodilator or LABA/ICS combination. ' Evidence exists for the superior efficacy of triple therapy compared with LABA/ICS and LAMA monotherapy with regards to improved lung function, health status, and exacerbation rate. However, the benefits of triple therapy compared with dual bronchodilation (LABA/LAMA) are uncertain. ${ }^{2}$ The IMPACT study compared triple therapy and dual inhaler therapy comprising LABA/LAMA in patients with COPD. ${ }^{3}$ In an expert interview, Dr Cazzola discusses this study and its implications for clinical practice.

\section{Q. What are the limitations of dual therapy in COPD?}

I advocate the need to start treatment of COPD as soon as the diagnosis is made. LABA/LAMA (dual bronchodilation) should be employed in order to optimise bronchodilation. After having maximized bronchodilator treatment with LABA/LAMA regimens, and only in patients with more symptomatic GOLD group D COPD, treatment can be escalated to triple therapy which either adds an ICS to dual-bronchodilator therapy, or a LAMA to existing LABA/ICS. However, the real-life evidence shows that even pulmonologists often prefer to use triple therapy even in patients who are not suffering from severe COPD. This prescriptive behaviour is likely due to the confidence that physicians have in starting a full treatment to ensure the best care to their patients.

\section{Q. Could you tell us a little about the recent IMPACT study comparing triple and dual therapy in COPD?}

The IMPACT study was a phase III, randomised, double-blind, triple-arm, parallel-group, global multicentre study comparing the rate of moderate and severe exacerbations between umeclidinium/vilanterol/fluticasone furoate (UMEC/VI/FLF) and VI/FLF or UMEC/VI in 10,355 patients with COPD over a 52-week treatment period. ${ }^{3}$ Inclusion criteria were patients with either a forced expiratory volume in 1 second $\left(\mathrm{FEV}_{1}\right.$ ) less than $50 \%$ of the predicted normal value and a history of at least one moderate or severe exacerbation in the previous year, or an $\mathrm{FEV}_{1}$ of $50-80 \%$ of the predicted normal value and at least two moderate exacerbations or one severe exacerbation in the previous year. There was a significant reduction in moderate-to-severe exacerbation rates with triple therapy (-15\% versus VI/FLF and $-25 \%$ versus UMEC/VI). The rate of moderate or severe exacerbations in the UMEC/VI/FLF group was 0.91 per year, as compared with 1.07 per year in the 
VI/FLF group (rate ratio, 0.85) and 1.21 per year in the UMEC/VI group (rate ratio, 0.75). Triple therapy also significantly reduced the annual rate of severe exacerbations compared with UMEC/VI (rate ratio, 0.66; $34 \%$ difference), but not with VI/FLF (rate ratio, 0.87; 13\% difference). UMEC/VI/FLF was more effective than the other two treatments in improving the trough $\mathrm{FEV}_{1}$ at week $52(+97 \mathrm{~mL}$ versus $\mathrm{VI} / \mathrm{FF}$, and $+54 \mathrm{~mL}$ versus UMEC/VI). It was also significantly better than the other two treatments with respect to the impact on St George's Respiratory Questionnaire (SGRQ) total score and in the percentage of patients who had a response as defined by a decrease in the SGRQ total score of at least 4 points. Furthermore, in a subset of 5,058 patients, the percentage of patients who had a response as defined by an increase in the Transition Dyspnoea Index of at least 1 unit was higher with triple therapy than with either dual therapy. The risk of clinician-diagnosed pneumonia was significantly higher with UMEC/VI/FLF than UMEC/VI as assessed in a time-to-first-event analysis (hazard ratio, 1.53), but not than VI/FLF (hazard ratio, 1.02).

\section{Q. What were the strengths and limitations of this study?}

Suissa and Drazen, commenting on the IMPACT study data, ${ }^{4}$ highlighted that although this trial has addressed the possibility of a step-up approach from a dual long-acting bronchodilator regimen to triple therapy, the results of the study were probably artificially inflated because the majority of the enrolled patients were already treated with ICSS and some of them had a history of asthma. For this reason, they think that the IMPACT trial falls short of providing the anticipated strong evidence to better understand the potential for stepping-up to single inhaler triple therapy in clinical practice. In any case, UMEC/VI/FLF and VI/FLF also showed a signal toward lower all-cause mortality during treatment than UMEC/VI. However, we do not believe that it is an outcome endowed with consistency, and this not only because it contradicts the results of the SUMMIT study that was powered to evaluate all-cause mortality and did not show a significant effect for $\mathrm{VI} / \mathrm{FLF}^{5}$ but also, and mainly because death from any cause during treatment was not a prespecified primary and secondary outcome. This means that the study was not appropriately powered to assess effects on mortality.

\section{Q. How are these findings likely to impact on clinical practice with COPD in the short and long term?}

Despite these limitations, we fully agree that the available evidence also produced by other trials, such as FULFIL ${ }^{6}$ and TRIBUTE, ${ }^{7}$ does support the current recommendation of triple therapy for GOLD D patients. It is likely that the fundamental question in the next treatment paradigm of COPD will no longer be whether and/or when it is appropriate to switch patients with COPD from a LABA/ICS regimen to a LAMA/LABA one, but rather in which patients and when we can add an ICS to the dual bronchodilation. I strongly believe that maximising the treatment in patients with a degree of clinical instability by including an ICS in the therapeutic regimen is useful to control the disease, but may not be needed during periods of clinical stability. However, it is always better to avoid a therapeutic step-up progression when it is not needed rather than being forced subsequently into a step-down approach in which the outcome is always unpredictable.

\section{Q. What (if any) further evidence is needed to support the routine use of triple therapy in COPD?}

We have carried out a treatment comparison by systematic review and quantitative synthesis on the available clinical evidence, with analyses incorporating data from studies that have compared triple therapy with double bronchodilation and/or single bronchodilator therapy (submitted). Preliminary data indicate that triple therapy is significantly more effective than double bronchodilation and single bronchodilator therapy in reducing the risk of moderate or severe exacerbations. It induces a numerically greater increase in the value of trough $\mathrm{FEV}_{1}$ compared with single bronchodilator therapy rather than with double bronchodilation, and the cumulative meta-analysis of the subgroups showed that the protective effect of triple therapy compared with double bronchodilation relative to the risk of moderate or severe exacerbation became greater in patients with higher eosinophil counts in the blood in a convincing stepwise fashion. In any case, meta-analyses deal with populations, not with single individuals. Thus, clinicians must use prudence when applying the conclusions derived from this investigative technique to the individual patient. For this reason, we are waiting for the results of the ETHOS study (NCT02465567), a randomised, double-blind, multi-centre, parallelgroup study that is assessing the efficacy and safety of glycopyrronium bromide/formoterol fumarate/budesonide (GB/FF/BUD) relative to GB/FF 14.4/9.6 $\mu \mathrm{g}$ and FF/BUD 9.6/320 $\mu \mathrm{g}$ on acute exacerbations of COPD over a 52-week treatment period in approximately 8,000 patients with moderate to very severe COPD, mainly because of its number of patients studied. However, we must highlight that the step-up approach from dual bronchodilation to triple therapy takes no account of the critical differences in COPD exacerbations (they differ in aetiology, severity, and biological substrate), and thus it is not designed on the patient's specific needs to be treated. This is an important limitation if we aim to personalise the therapy of our patients.
1. Global Initiative for Asthma (GINA). 2018 GINA Report. Global Strategy for Asthma Management and Prevention. Available at: https://ginasthma.org/2018-gina-report-globalstrategy-for-asthma-management-and-prevention/ (accessed 20 August 2018)

2. Fabbri LM, Roversi S, Beghe B. Triple therapy for symptomatic patients with COPD. Lancet. 2017;389:1864-5. Lipson DA, Barnhart F, Brealey N, et al. Once-daily single-inhaler triple versus dual therapy in patients with COPD. $N$ Eng/ I Med. 2018;378:1671-80.

4. Suissa S, Drazen JM. Making sense of triple inhaled therapy for COPD. N Eng/ J Med. 2018;378:1723-4.

5. Vestbo J, Anderson JA, Brook RD, et al. Fluticasone furoate and vilanterol and survival in chronic obstructive pulmonary disease with heightened cardiovascular risk (SUMMIT): a double-blind randomised controlled trial. Lancet. 2016;387:1817-26.
6. Lipson DA, Barnacle H, Birk R, et al. FULFIL trial: once-daily triple therapy for patients with chronic obstructive pulmonary disease. Am I Respir Crit Care Med. 2017;196:438-46.

7. Papi A, Vestbo J, Fabbri L, et al. Extrafine inhaled triple therapy versus dual bronchodilator therapy in chron obstructive pulmon blind, parallel group, randomised controlled trial. Lancet. 2018;391:1076-84. 